\title{
Correction to: Binding of silver nanowaste using jellyfish immune reaction extract and an assessment of aquatic toxicity
}

\author{
Euna $\mathrm{Kim}^{1} \cdot$ Min-Kyeong Yeo ${ }^{1,2} \cdot$ Bong Gu Lee ${ }^{1} \cdot$ Sun Woo Geum ${ }^{1}$
}

Published online: 28 January 2022

(c) The Author(s) under exclusive licence to The Korean Society of Toxicogenomics and Toxicoproteomics 2022

Correction to: Mol. Cell. Toxicol.

https://doi.org/10.1007/s13273-021-00199-6

Due to an unfortunate oversight during the e.proofing, Table 3 has been given erroneously. It should be read:

Table 3 Total amount of living bacteria $1 \mathrm{~h}$ after exposure to the experimental groups

\begin{tabular}{lll}
\hline & Ct value & Copies $/ \mathrm{mL}$ \\
\hline Control & $18.51 \pm 0.42$ & $2.18 \times 10^{6}$ \\
BJEI & $22.41 \pm 0.89$ & $1.39 \times 10^{5 * *}$ \\
AgN-Citrate & $18.97 \pm 1.45$ & $1.58 \times 10^{6}$ \\
AgN-PVP & $19.15 \pm 0.48$ & $1.39 \times 10^{6}$ \\
AgN-Citrate+BJEI & $24.40 \pm 2.07$ & $3.38 \times 10^{4 * *}$ \\
AgN-PVP+BJEI & $24.85 \pm 2.22$ & $2.45 \times 10^{4 * *}$ \\
\hline
\end{tabular}

**Indicates significant differences in comparison to the control $(p<0.01)$
The original article has been corrected.

Publisher's Note Springer Nature remains neutral with regard to jurisdictional claims in published maps and institutional affiliations.

The original article can be found online at https://doi.org/10.1007/ s13273-021-00199-6.

Min-Kyeong Yeo

bioclass@khu.ac.kr

1 Department of Applied Environmental Science, Graduate

School, Kyung Hee University, 1732 Deogyeong-daero,

Giheung-gu, Yongin-si, Gyeonggi-do 17104,

Republic of Korea

2 Department of Environmental Science and Engineering,

College of Engineering, Kyung Hee University,

1732 Deogyeong-daero, Giheung-gu, Yongin-si,

Gyeonggi-do 17104, Republic of Korea 